\title{
Self-Sustained Kinetic Oscillations in CO Oxidation over Silica-Supported Pt
}

\author{
Per-Anders Carlsson, ${ }^{1, *}$ Vladimir P. Zhdanov, ${ }^{1,2}$ and Magnus Skoglundh ${ }^{1,3}$ \\ ${ }^{1}$ Competence Centre for Catalysis, Chalmers University of Technology, SE-412 96 Göteborg, Sweden \\ ${ }^{2}$ Boreskov Institute of Catalysis, Russian Academy of Sciences, Novosibirsk 630090, Russia \\ ${ }^{3}$ Applied Surface Chemistry, Chalmers University of Technology, SE-412 96 Göteborg, Sweden
}

(Dated: April 5, 2004)

\begin{abstract}
Isothermal self-sustained kinetic oscillations in $\mathrm{CO}$ oxidation over silica-supported $\mathrm{Pt}$ at nearatmospheric pressure were studied by combined in situ Fourier transformed infrared spectroscopy and mass spectrometry. The use of a specially designed reactor and careful choice of the physical properties of the catalyst and reaction conditions made it possible to eliminate diffusion limitations, to determine the maximum $\mathrm{CO}$ oxidation rate per $\mathrm{Pt}$ site in the purely kinetic regime and to clarify the mechanism of the oscillations. Specifically, our results indicate that during the high reactive periods the reaction mainly occurs on the oxide surface.
\end{abstract}

The understanding of the kinetics of heterogeneous catalytic reactions is of high interest, since heterogeneous catalysis is the mainstay of the chemical industry [1]. In practice, these reactions often occur on nm-sized crystallites deposited on porous support materials. Experimental studies of catalytic reactions are traditionally based on the use of sophisticated physical methods developed in surface science and solid state physics. Thus, heterogeneous catalysis has evolved into a fascinating interdisciplinary branch of natural sciences. Besides chemists, chemical engineers and physicists working in the fields mentioned above, the complex kinetics of catalytic reactions attract appreciable attention also amongst experts in non-linear science. In this communication, all these aspects are illustrated by providing new insight on the oscillatory kinetics of $\mathrm{CO}$ oxidation on supported Pt.

Since Langmuir's pioneering studies [2], the oxidation of $\mathrm{CO}$ over $\mathrm{Pt}$ is a classical example of a heterogeneous catalytic reaction. It is considered to be generic due to its apparently simple mechanism, richness of spatiotemporal behavior, and practical relevance [3]. Kinetic oscillations in this reaction were first found by Hugo in 1970 [4] on a supported catalyst. Later on, this phenomenon was observed also for other types of catalysts (polycrystalline wires and single crystals) both at ultrahigh vacuum (UHV) and subatmospheric conditions [3]. In particular, Ertl and co-workers [see Ref. [5] and review [3](b)] have demonstrated that on $\operatorname{Pt}(100)$ and (110), at $\mathrm{UHV}$, the oscillations result from the interplay between bistability and adsorbate-induced surface reconstruction exposing patches with different $\mathrm{O}_{2}$ sticking probabilities. In other situations and especially at subatmospheric conditions (this practically important case is discussed below), the mechanism of oscillations is still open for debate. The main tendency here is to relate oscillations with the formation of surface oxides. The first oxide model, suggested by Sales et al. [6] for a Pt wire, implies that the reaction occurs primarily via the conventional Langmuir-Hinshelwood mechanism on the unoxidised sites. For this scheme (or for reaction schemes including adsorbate-induced surface restructuring), one could expect that during the high reactive periods the maximum reaction rate would be of the order of or about one order of magnitude lower than the $\mathrm{CO}$ impingement rate, because the reaction rate is controlled in this situation by the interplay between the $\mathrm{CO}$ and $\mathrm{O}_{2}$ adsorption rates and the sticking coefficient for $\mathrm{CO}$ adsorption on such sites is high. An analysis of the kinetic parameters used in Ref. [6] indicates however that this was not the case [7]. Thus, the model proposed in Ref. [6] does not seem to be self-consistent. More recent STM-based studies [8] of bistability of $\mathrm{CO}$ oxidation on $\mathrm{Pt}(110)$ inside a micro-flow reactor show that the reaction does involve the oxide formation but in the high reactive state it actually occurs on the oxide surface. The latter seems to explain why the reaction rate during the high reactive periods is low compared to what one could expect. The reports of oscillations in $\mathrm{CO}$ oxidation observed for supported $\mathrm{Pt}$ in catalytic reactors are numerous $[4,9]$, but the physics behind these oscillations is still not clear. Partly, this is connected with the limitations of in situ methods to experimentally study surface reactions in porous catalysts. In addition, as a rule [except Refs. [9] $(\mathrm{a}, \mathrm{d}, \mathrm{i}, \mathrm{j})]$, there is no guarantee that the reaction conditions in a reactor are isothermal and that during the high reactive periods the reaction is not influenced by external or internal diffusion of reactants. For this reason, to our knowledge, the accurate estimation of the maximum $\mathrm{CO}$ oxidation rate per Pt site is still lacking. Our goal was to perform the corresponding measurements in combination with transient in situ Fourier transformed infrared (FTIR) spectroscopy in order to clarify the mechanism of oscillations.

The silica supported Pt sample was prepared according to Ref. [10] by impregnating silica with a halogenfree platinum precursor. Colloidal silica (Bindizil 40 NH3 170, $20 \mathrm{~nm}$ spherical particles, Akzo Nobel) was dispersed in distilled water, and the $\mathrm{pH}$ was adjusted to 10.5 by ammonium hydroxide (25\% $\mathrm{NH}_{4}$, Merck). An aqueous solution of $\mathrm{Pt}\left(\mathrm{NH}_{3}\right)_{4}(\mathrm{OH})_{2}$ (Johnsson Matthey) was then added dropwise to the silica sol under continuous stirring. The sol was thereafter instantly frozen with liquid nitrogen and freeze-dried. The resulting powder was cal- 
cined in air at $873 \mathrm{~K}$ for $1.5 \mathrm{~h}$. The chemisorbed amount of CO (Micromeritics ASAP2010C) was $7.2 \mu \mathrm{mol} / \mathrm{g} \mathrm{sam-}$ ple which corresponds to a $\mathrm{Pt}$ dispersion of about $20 \%$ and a mean diameter of $5.7 \mathrm{~nm}$ assuming spherical particles and 0.7 CO molecules chemisorbed per surface $\mathrm{Pt}$ atom [11]. The BET surface area was measured to be $132 \mathrm{~m}^{2} / \mathrm{g}$ and the particle diameter of the sample was less than $50 \mu \mathrm{m}$ (SEM analysis).

The time-resolved in situ FTIR spectroscopy experiments (Fig. 1) were performed in diffuse reflectance mode with a BioRad FTS6000 spectrometer equipped with a Harrick Praying Mantis DRIFT cell. The product stream was continuously analysed with a Baltzer quadropole mass spectrometer. Collection of eight IR spectra (Kubelka-Munk mode) per second keeping a wavenumber resolution of $1.2 \mathrm{~cm}^{-1}$ was possible using a nitrogen cooled MCT detector. The reactor with the diameter $D=6 \mathrm{~mm}$ and length $L=3 \mathrm{~mm}$ was loaded with $50 \mathrm{mg}$ powder mixture containing $9.1 \mathrm{wt} .-\%$ catalyst diluted in $\mathrm{KBr}$. The mixture was pre-treated with 5 vol.- $\% \mathrm{O}_{2}$ in $\mathrm{Ar}$ at $823 \mathrm{~K}$ for $10 \mathrm{~min}$ and then dried at $423 \mathrm{~K}$ for $12 \mathrm{~h}$ and a reference spectrum was collected. The sample was then exposed to 1000 vol.-ppm CO in Ar. Each experiment was performed at $423 \mathrm{~K}$ using a total flow of $300 \mathrm{ml}(\mathrm{NTP}) / \mathrm{min}$.

The small size of the reactor and the high flux of the reactants are the main advantages of our experimental setup compared to those used in earlier studies [4] and $[9](b-j)$. These two factors combined with sufficiently small porous catalyst particles, make it possible to avoid (see below) both internal and external diffusion limitations.

From Fig. 1, it is evident that during oscillations the adsorbate composition on the Pt surface changes significantly. The large variation in intensity of the band for linearly-bonded $\mathrm{CO}\left[\tilde{\nu}_{\text {lin }}^{C O}(\mathrm{Pt})\right]$ at around $2090 \mathrm{~cm}^{-1}$ (top panels) corresponds to a $\mathrm{CO}$ coverage oscillating between 0.9 and near zero (the unit $\mathrm{CO}$ coverage corresponds here to the equilibrium coverage for a 1000 vol.-ppm $\mathrm{CO} / \mathrm{Ar}$ feed at $423 \mathrm{~K}$ ). The transitions between high and low $\mathrm{CO}$ coverage clearly demonstrate the bistable kinetics exhibiting two distinctly different reaction regimes. One with high CO coverage for which the reaction proceeds in the so-called $\mathrm{CO}$ self-poisoned regime with a relatively low rate and low $\mathrm{CO}_{2}$ production (of about 120 vol.$\mathrm{ppm}$ ), and another one with low $\mathrm{CO}$ coverage for which the reaction rate is appreciably higher (resulting in about 250 vol.-ppm $\mathrm{CO}_{2}$ ).

An interesting observation is the multiple oscillation frequencies of the $\tilde{\nu}_{\text {lin }}^{C O}(\mathrm{Pt})$ and $\tilde{\nu}_{\text {gas }}^{\mathrm{CO}_{2}}$ bands, which oscillate in counter phase, i.e., both slowly (of the order of minutes) and more rapidly (of the order of seconds) in an almost fluctuating manner. Obviously, the rapid oscillations are not captured by the MS response signal for $\mathrm{CO}_{2}$. At present, we cannot unambiguously state whether this discrepancy is due a limited IR beam penetration depth, i.e., only a sub-volume of the catalyst bed is viewed and thus local fluctuations in the $\mathrm{CO}$ coverage may have a large impact on the overall IR intensity, while the MS response signals naturally refer to the entire catalyst bed, or if the frequency of these rapid oscillations is too high to be resolved by our MS configuration, i.e., unsuitable piping.

Scrutinizing the IR panels, a hysteresis-like behavior in the intensity for the $\tilde{\nu}_{\text {lin }}^{C O}(\mathrm{Pt})$ band, and correspondingly the intensity of the $\tilde{\nu}_{\text {gas }}^{C O_{2}}$ band, during oscillations can be discerned. The transition from the CO self-poisoned regime to the high-reactive regime is very fast (less than $0.2 \mathrm{~s}$ ) while the reverse transition occurs over a time period of about $10 \mathrm{~s}$. Furthermore, during the phases where the $\mathrm{CO}$ coverage builds up, a small blue-shift of the $\tilde{\nu}_{\text {lin }}^{C O}(\mathrm{Pt})$ band of about $6 \mathrm{~cm}^{-1}$ towards higher wavenumbers occurs. This is the anticipated frequency shift due to repulsive CO-CO lateral interactions with increasing $\mathrm{CO}$ coverage.

The analysis indicates (see Appendix) that the oscillatory kinetics observed in our experiments are not influenced by diffusion limitations. In this case, the $\mathrm{CO}$ impingement rate per site is defined by $F=$ $N_{A} a_{s} p_{C O} / \sqrt{2 \pi M R T}$, where $N_{A}=6.02 \cdot 10^{23} 1 / \mathrm{mol}$ is the Avogadro constant, $R=8.31 \mathrm{~J} /(\mathrm{mol} \mathrm{K})$ the molar gas constant, $a_{s}=8.0 \cdot 10^{-20} \mathrm{~m}^{2}$ the area of a Pt site, $M=28 \cdot 10^{-3} \mathrm{~kg} / \mathrm{mol}$ the $\mathrm{CO}$ molecular weight, and $P_{C O}=100 \mathrm{~Pa}$ the bulk $\mathrm{CO}$ pressure. With these values at $T=423 \mathrm{~K}$, one obtains $F=1.94 \cdot 10^{5}$ molecules $/($ site s). The observed maximum reaction rate per site is given by $r_{o b s}=q p_{C O} X /\left(R T n_{s}\right)$, where $q=5.0 \cdot 10^{-6} \mathrm{~m}^{3} / \mathrm{s}$ is the volumetric flow rate at room temperature, $X=0.25$ the $\mathrm{CO}$ conversion, and $n_{s}=W[\mathrm{Pt}$ loading $]\left[\mathrm{Pt}\right.$ dispersion] $/ M_{P t}$ the number of sites $\left(W=4.6 \cdot 10^{-6} \mathrm{~kg}\right.$ is the amount of catalyst, and $M_{P t}=0.195 \mathrm{~kg} / \mathrm{mol}$ is the Pt molecular weight). With the $\mathrm{Pt}$ loading $=0.01$ and $\mathrm{Pt}$ dispersion $=0.20$, the reaction rate is $r_{o b s}=1.1$ molecules/(site s), and accordingly the ratio between the impingement rate and the reaction rate is $F / r_{\text {obs }}=1.94 \cdot 10^{5} / 1.1 \approx 2 \cdot 10^{5}$. The high value of this ratio indicates that during the high reactive periods the reaction hardly occurs via the conventional Langmuir-Hinshelwood mechanism.

As already mentioned, the kinetic oscillations in $\mathrm{CO}$ oxidation on supported $\mathrm{Pt}$ are often assumed to be related with surface-oxide formation. In particular, the oxide mechanism proposed by Sales et al. [6] was believed to be operative in Refs. [9] $(\mathrm{d}, \mathrm{g})$ (in other references, e.g. in Ref. [9](h), the mechanistic details of the influence of oxide on the reaction are not specified). On alumina, for example, Lindstrom et al. [9](d,e) found an oscillating IR absorption band at around $2120 \mathrm{~cm}^{-1}$ which they attributed to linearly bonded $\mathrm{CO}$ on electron deficient $\mathrm{Pt}$ $\left[\tilde{\nu}_{l i n}^{C O}\left(\mathrm{Pt}^{+}\right)\right]$, i.e., platinum oxide. However, in the literature one can find alternative mechanistic explanations for oscillations $[9](\mathrm{a}, \mathrm{f}, \mathrm{j})$. In the most detailed study, Fan- 

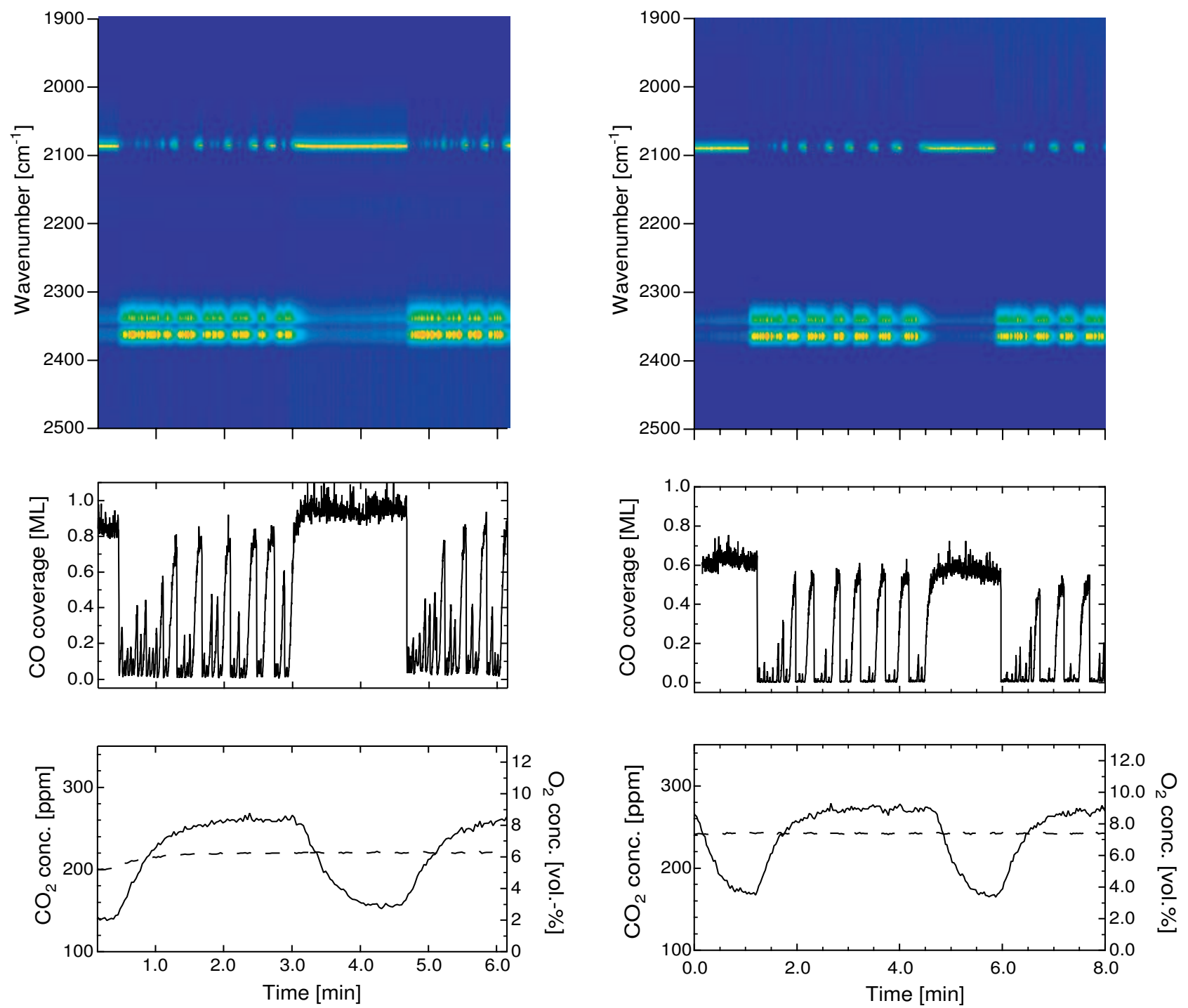

FIG. 1: Simultaneous FTIR spectroscopy and MS analysis of isothermal kinetic oscillations in oxidation of 1000 vol.-ppm CO over $1 \% \mathrm{Pt} / \mathrm{SiO}_{2}$ at $423 \mathrm{~K}$ with 6.0 vol.- $\%$ (left side) and 7.0 vol.- $\% \mathrm{O}_{2}$ in Ar (right side), respectively. The top panels display a two dimensional representation of the vibrational stretching region for linearly bonded $\mathrm{CO}\left[\tilde{\nu}_{l i n}^{C O}(\mathrm{Pt})\right]$ at around $2090 \mathrm{~cm}^{-1}$ and gaseous $\mathrm{CO}_{2}\left[\tilde{\nu}_{\text {gas }}^{\mathrm{CO}_{2}}\right]$ at around $2350 \mathrm{~cm}^{-1}$ (bright colors correspond to high IR intensity). The middle panels show $\mathrm{CO}$ coverage obtained by integrating the $\tilde{\nu}_{\text {lin }}^{C O}(\mathrm{Pt})$ IR band (the unit CO coverage corresponds to the equilibrium coverage for a 1000 vol.-ppm $\mathrm{CO} / \mathrm{Ar}$ feed at $423 \mathrm{~K}$ ). The bottom panels represent $\mathrm{CO}_{2}$ and $\mathrm{O}_{2}$ concentrations (solid and dashed lines, respectively) for the product stream. On the horizontal axis, $t=0$ corresponds to the moment after about 40 min of observation of self-sustained oscillations.

son et al. $[9](\mathrm{j})$ investigated oscillations over $\mathrm{Pt} / \mathrm{SiO}_{2}$ at 1-10 Torr at 450-570 K by combined FTIR spectroscopy and MS and found no evidence for $\tilde{\nu}_{\text {lin }}^{C O}\left(\mathrm{Pt}^{+}\right)$. Instead, they attributed the oscillations to $\mathrm{CO}$ island formation, like during adsorbate-induced surface restructuring of $\mathrm{Pt}(100)$ and (110), since the stretching frequency of linearly bonded $\mathrm{CO}$ on $\mathrm{Pt}\left[\tilde{\nu}_{\text {lin }}^{C O}(\mathrm{Pt})\right.$ at about $\left.2080 \mathrm{~cm}^{-1}\right]$ was nearly constant in the course of oscillations (CO island formation and $\mathrm{Pt}(110)$ restructuring were proposed also by other authors $[9](\mathrm{a}, \mathrm{f}))$. The mechanism including island formation was supported by complementary isotope mixing experiments.

Similar to previous studies $[9](\mathrm{a}, \mathrm{f}, \mathrm{j})$, the main oscillations in our reactor occur on a time scale of the order of minutes and with minor change of the frequency position of the $\tilde{\nu}_{\text {lin }}^{C O}(\mathrm{Pt})$ band during the oscillations. We observe, however, significantly higher $\mathrm{CO}$ coverage, up to 0.8-0.9 compared to below 0.3 as reported by Fanson et al. $[9](\mathrm{j})$. This may indicate different feedback mechanisms in these studies. Despite the fact that neither we nor other authors $[9](\mathrm{f}, \mathrm{j})$ were able to clearly detect a $\tilde{\nu}_{\text {lin }}^{C O}\left(\mathrm{Pt}^{+}\right)$band, we believe that the mechanism includ- 
ing surface restructuring is unlikely, because this mechanism implies that during the high reactive periods the reaction occurs primarily via the conventional LangmuirHinshelwood mechanism and this is not in line with the results of our measurements. In our study, the $\tilde{\nu}_{\text {lin }}^{C O}(\mathrm{Pt})$ band is positioned at $2090 \mathrm{~cm}^{-1}$ which is very close to the value of $2092 \mathrm{~cm}^{-1}$ reported for $\mathrm{CO}$ adsorbed on an oxidised $\mathrm{Pt} / \mathrm{SiO}_{2}$ catalyst with a $\mathrm{Pt}$ dispersion similar to our sample $[9](\mathrm{j})$. This indicates that we instead may assign this IR band to CO adsorbed on partially oxidised $\mathrm{Pt}\left[\tilde{\nu}_{\text {lin }}^{C O}\left(\mathrm{Pt}^{\delta+}\right)\right]$, like it was assigned in our previous study [12] of the non-oscillatory kinetics. Thus, the oxide seems to be present also when the $\mathrm{CO}$ coverage is relatively high. Moreover, the slow build up of the CO coverage during the transition from the high to the low reaction rate branch (the hysteresis) may be due to simultaneous CO adsorption and oxide reduction.

In summary, we have carefully studied isothermal selfsustained kinetic oscillations in CO oxidation over silicasupported $\mathrm{Pt}$ in a reactor at near-atmospheric pressure in the absence of diffusion limitations. The MS results obtained demonstrate that during the high reactive periods the $\mathrm{CO}$ impingement rate is about five orders of magnitude higher than the reaction rate. This finding in combination with our FTIR spectroscopy data favour a model including the formation of oxide islands where (i) $\mathrm{CO}$ is primarily adsorbed outside these islands and (ii) during the high reactive periods the reaction mainly occurs on the oxide surface [for oscillations in CO oxidation on $\operatorname{Pd}(100)$, the latter was demonstrated in Ref. [8](b)].

This work has been performed within the Competence Centre for Catalysis, which is hosted by Chalmers University of Technology and financially supported by the Swedish Energy Agency and the member companies: AB Volvo, GM Powertrain Sweden AB, Volvo Car Corporation, Scania CV AB, Perstorp Specialty Chemicals AB, Haldor Topsøe A/S, Albemarle Catalysts BV and the Swedish Space Corporation.

\section{Appendix}

The role of external diffusion limitations can be estimated by comparing the concentration difference of $\mathrm{CO}$ over the gas film $\left(\Delta C_{C O}\right)$ with the bulk concentration of $\mathrm{CO}\left(C_{C O}^{b}\right)$. First, we note that the observed reaction rate per unit mass of catalyst is approximately given by $r_{o b s}=q p_{C O} X /(R T W)$, where $q=5.0 \cdot 10^{-6} \mathrm{~m}^{3} / \mathrm{s}$ is the volumetric flow rate at room temperature, $p_{C O}=100 \mathrm{~Pa}$ is the partial CO pressure, $T=423 \mathrm{~K}$ is temperature, $X=0.25$ is the CO conversion, and $W=4.6 \cdot 10^{-6} \mathrm{~kg}$ is the amount of catalyst. With these values, one obtains $r_{\text {obs }}=11.0 \cdot 10^{-3} \mathrm{~mol} /$ ( $\mathrm{s} \mathrm{kg}$ catalyst $)$.

For a packed-bed reactor with spherical porous catalyst particles, the diffusion-controlled reaction rate is $r=S_{m} k_{c} \Delta C_{A}$, where $S_{m}=6 /\left(d_{p} \rho_{p}\right)$ is the external surface area per unit mass of catalyst, $d_{p}=50 \cdot 10^{-6} \mathrm{~m}$ and $\rho_{p}=1.5 \cdot 10^{3} \mathrm{~kg} / \mathrm{m}^{3}$ are the diameter and density of the porous catalyst particles, and $k_{c}=S h D_{A B} / d_{p}$ is the mass transfer coefficient ( $S h$ is the Sherwood number and $D_{A B}=3.7 \cdot 10^{-5} \mathrm{~m}^{2} / \mathrm{s}$ is the binary gas diffusivity). Expressing $\Delta C_{A}$ via $r$ yields $\Delta C_{A}=r d_{p}^{2} \rho_{p} /\left(6 S h D_{A B}\right)$. Substituting into the latter expression $r_{o b s}$ for $r$ and using a low value for the Sherwood number, $S h=1.5$ (this value overestimates the concentration gradient), we get $\Delta C_{A}=1.3 \cdot 10^{-4} \mathrm{~mol} / \mathrm{m}^{3}$, which is minor compared to the bulk $\mathrm{CO}$ concentration, $C_{A}^{b}=p_{A} /(R T)=$ $2.8 \cdot 10^{-2} \mathrm{~mol} / \mathrm{m}^{3}$, at $\mathrm{T}=423 \mathrm{~K}$.

The influence of internal diffusion limitations in a porous catalyst can be estimated by calculating the Weisz modulus, $\Phi=r_{v} d_{p}^{2} /\left(4 C_{C O}^{s} D_{e f f}\right)$, where $r_{v}$ is the reaction rate per unit volume of catalyst, $C_{C O}^{s}$ the $\mathrm{CO}$ concentration at the surface of the pellet, and $D_{\text {eff }}=$ $4.7 \cdot 10^{-7} \mathrm{~m}^{2} / \mathrm{s}$ the effective diffusivity. The internal concentration gradients are negligible at $\Phi<1$. Since the external diffusion limitations are minor (see above), the concentration at the surface of a catalyst particle is well approximated with the concentration in the gas bulk, and we have $\Phi=r_{o b s} \rho_{c a t} d_{p}^{2} /\left(4 C_{C O}^{b} D_{\text {eff }}\right)$. Substituting the specific values of the parameters into the latter expression yields $\Phi \approx 0.77<1$. Thus, the influence of internal diffusion limitations are negligible.

* perc@chemeng. chalmers.se

[1] J.M. Thomas and W.J. Thomas, Principles and Practice of Heterogeneous Catalysis, VCH, Weinheim, 1997.

[2] I. Langmuir, Trans. Farad. Soc., 1922, 17, 672.

[3] (a) F. Schüth et al., Adv. Catal., 1993, 39, 51; (b) R. Imbihl and G. Ertl, Chem. Rev., 1995, 95, 697; (c) H.H. Rotermund, Surf. Sci. Rep., 1997, 29, 265; (d) V.P. Zhdanov, Surf. Sci. Rep., 2002, 45, 231.

[4] P. Hugo, Ber. Bunsenges. Phys. Chem., 1970, 74, 121.

[5] G. Ertl et al., Phys. Rev. Lett., 1982, 49, 177.

[6] B.C. Sales et al., Surf. Sci., 1982, 114, 381.

[7] P.-A. Carlsson et al., Appl. Surf. Sci., 2005, 239, 424.

[8] B.L.M. Hendriksen et al., (a) Phys. Rev. Lett., 2002, 89, 046101; ibid. 2005, 95, 255505; (b) Catal. Today, 2005, 105, 234.

[9] (a) E. Wicke et al., Ber. Bunseges. Phys. Chem., 1980, 84, 315; (b) D.T. Lynch and S.E. Wanke, J. Catal., 1984, 88, 333; (c) D.J. Kaul and E.E. Wolf, J. Catal., 1985, 91, 216. (d) T.H. Lindstrom and T.T. Tsotsis, Surf. Sci., 1986, 171, 349; (e) T.H. Lindstrom and T.T. Tsotsis Surf. Sci., 1985, 150, 487; (f) F. Schüth and E. Wicke, Ber. Bunseges. Phys. Chem., 1989, 93, 191; (g) N. Hartmann et al., Catal. Lett., 1994, 28, 373; (h) F. van Neer and A. Bliek, Chem. Eng. Sci., 1999, 54, 4483; (i) J. Lauterbach et al. Chem. Eng. Sci., 1999, 54, 4501; (j) P.T. Fanson et al. J. Catal., 2001, 204, 35.

[10] I.-M. Axelsson et al., Appl. Catal., 1988, 44, 251.

[11] P. Lööf et al., J. Catal., 1991, 130, 181.

[12] P.-A. Carlsson et al., J. Catal., 2004, 226, 422. 